\title{
Associative processes controlling the persistence of operant responding: S-S* and R-S*
}

\author{
ROGER L. MELLGREN and MARK W. OLSON \\ University of Oklahoma, Norman, Oklahoma 73019
}

\begin{abstract}
Rats were trained initially to barpress for food when either a tone or a light was presented. One group then had the tone-food $\left(\mathbf{S}_{\mathrm{T}}-\mathrm{S}^{*}\right)$ association extinguished by presentation of the tone in the absence of the bar followed by no food. Under this condition, the response-food (R-S*) association was assumed to remain intact. Another group had the R-S* but not the tone-food $\left(\mathrm{S}_{\mathrm{T}}-\mathrm{S}^{*}\right)$ association extinguished by presentation of the light when a barpress did not produce a food pellet. Two control groups, one concerned with generalized extinction and the other receiving no treatment, were included. The persistence of responding to the tone was then measured in extinction. Extinguishing either type of association $\left(\mathbf{S}_{\mathrm{T}}-\mathrm{S}^{*}\right.$ or $\left.\mathrm{R}-\mathrm{S}^{*}\right)$ reduced resistance to extinction relative to controls, implicating both types of association as being important in determining the persistence of operant behavior, with the suggestion that the R-S* may be more important than the $\mathrm{S}_{\mathrm{T}} \mathrm{S}^{*}$. These results are inconsistent with the current trend of emphasizing the importance of $\mathrm{S}_{\mathrm{T}}-\mathrm{S}^{*}$ associations to the exclusion of other associative mechanisms.
\end{abstract}

Several experimental results have implicated S-S* associations as the most important associative process controlling operant behavior. The most influential procedure has been autoshaping or automaintenance (e.g., Brown \& Jenkins, 1968). The results of such experiments have been interpreted by Hearst and Jenkins (1974) and Moore (1973), among others, as showing the overriding importance of the stimulus-reinforcer (S-S*) association(s) in controlling behavior. Bindra (1978) goes so far as to claim that all learned behaviors are explained by S-S* associations, denying any explanatory importance of a response-reinforcer (R-S*) association.

The purpose of the current experiment was to evaluate the influence of S-S* and R-S* associations in determining the persistence of an operant response. In particular, the resistance to extinction of barpressing for rats was the dependent variable and the presumed manipulation of the strength of S-S* and R-S* associations was the independent variable. The use of resistance to extinction to test the influence of S-S* and R-S* association is important for a number of reasons. First, much of the theorizing concerning the nature of associative processes controlling operant behavior has been based on acquisition or maintenance of responding (i.e., autoshaping or automaintenance). Clearly, the persistence of a learned response in the face of altered circumstances is an equally important behavioral phenomenon. Second, extinction is a type of transfer-oftraining procedure, and separate-phase transfer-of-

Reprint requests should be addressed to Roger L. Mellgren, Department of Psychology, University of Oklahoma, Norman, Oklahoma 73019. Mark W. Olson is now at the University of Iowa. training paradigms have often been useful in determining what is learned in a particular situation. Finally, the extinction procedure, in particular, has been useful in assessing what is learned in acquisition and has received considerable theoretical attention (e.g., Amsel, 1967; Capaldi, 1967). The partial reinforcement effect is a good example of how extinction behavior reveals differences between experimental conditions that are not at all obvious during the acquisition or maintenance phase of the experiment.

The experiment consisted of three phases. In the first phase, rats were conditioned to press a bar to obtain a food pellet when a discrete signal was present (a tone or a light). We assumed that the rats would learn both $\mathrm{S}_{\mathrm{T}}-\mathrm{S}^{*}, \mathrm{~S}_{\mathrm{L}}-\mathrm{S}^{*}$ (tone-food, light-food) associations and a R-S* (barpress-food) association. In the second phase of the experiment, the strength of each association was manipulated. For one group, the $\mathrm{S}_{\mathrm{T}}-\mathrm{S}^{*}$ association was presumed to be weakened by presentation of the stimulus (tone) and no food pellet while the bar was absent from the operant chamber. For a second group, the bar remained in the chamber and the rat was presented the light, but barpressing was ineffective in producing the food pellet. For this group, the R-S* association was presumed to be weakened, but since the tone was never presented in this phase, we assumed that the $\mathrm{S}_{\mathrm{T}}-\mathrm{S}^{*}$ association was intact. Of course, the light-food, $\mathrm{S}_{\mathrm{L}}-\mathrm{S}^{*}$, association would also be weakened. It might be possible that $S_{L}-S^{*}$ extinction would generalize to the $\mathrm{S}_{\mathrm{T}} \mathrm{S}^{*}$ association. A control group was included to test this possibility. This control group experienced light/no food with the bar removed from the chamber. There should be no weakening of the R-S* association and any weakening of the $\mathrm{S}_{\mathrm{T}}-\mathrm{S}^{*}$ association should be due to generalization from the light to the tone. In 
order to assess this possibility, a second control group was simply put in the chamber during Phase 2 , with no bar present and no tone or light presented.

In the final phase of the experiment, all rats were presented the tone in extinction. One group was assumed to have the tone-food $\left(\mathrm{S}_{\mathrm{T}}-\mathrm{S}^{*}\right)$ association at full strength. The second group was assumed to have the R-S* association at low strength, but the $\mathrm{S}_{\mathrm{T}}-\mathrm{S}^{*}$ association at full strength. The two control groups were assumed to have both the $\mathrm{S}_{\mathrm{T}}-\mathrm{S}^{*}$ and R-S* associations at full strength. Thus the design of the experiment allows an assessment of the relative importance of the R-S* and S-S* associations in determining resistance to extinction of operant barpressing.

\section{METHOD}

\section{Subjects}

A total of 32 male albino rats, 180 days old at the start of the experiment, were randomly distributed into four groups of 8 each. The rats were obtained from the Holtzman Company, Madison, Wisconsin, and were experimentally naive.

\section{Apparatus}

Two identical operant conditioning chambers $25 \mathrm{~cm}$ square and $19 \mathrm{~cm}$ high were used. The bar, food cup, and lights were all located on one wall, the food cup in the center, the bar to the left, and the light (6-W jewel) to the right. A 4-in. speaker to deliver a tone $(1,000 \mathrm{~Hz})$ was located just outside the operant chamber, and the entire apparatus was enclosed in a ventilated sound-attenuating shell. Control of environmental events and recording of responses were accomplished by using standard $24-\mathrm{V}$ electromechanical devices located in a room adjoining the operant chambers.

\section{Procedure}

All subjects were deprived of food so that their ad-lib weights were reduced to $85 \%$ through controlled feedings that began 14 days before the start of the experiment and continued throughout. Each session was $48 \mathrm{~h}$ after the last, but feedings occurred every $24 \mathrm{~h}$.

In Phase 1 , the rats were all given training with light and tone as discrete signals for the availability of reinforcement (one 45-mg Noyes food pellet). On the first session, five pellets were placed in the food cup at the start of the session. A light or tone was presented $5 \mathrm{sec}$ after the subject was placed in the chamber, and a barpress response terminated the stimulus and delivered a food pellet. The next light or tone trial began $5 \mathrm{sec}$ after the delivery of the pellet unless another barpress response was made during the intertrial interval. An intertrial response postponed the next scheduled trial by $5 \mathrm{sec}$ on Session $1,10 \mathrm{sec}$ on Sessions 2 and 3 , and $20 \mathrm{sec}$ on Sessions 4-13. Once a trial began (i.e., a light or tone was presented), it continued until the rat pressed the bar. The programming of light and tone trials was such that there were equal numbers of each type trial in a nonsystematic order throughout the session. The duration of all sessions was $30 \mathrm{~min}$, and a total of 13 sessions were given in this phase.

In Phase 2, the groups received differential experiences with barpressing, lights, and tone. Group $\mathrm{S}_{\mathrm{T}}-\mathrm{S}^{*}$ received presentations of the light that were terminated when the subject pressed the bar or $10 \mathrm{sec}$ had elapsed. In either case, the termination of the light was accompanied by the operation of the empty pellet dispenser. Thus the designation of $\mathrm{S}_{\mathrm{T}}-\mathrm{S}^{*}$ refers to the association (torie-food) presumed to be intact at the end of Phase 2. Group R-S* received presentations of the tone that terminated with the operation of the empty pellet dispenser after $10 \mathrm{sec}$ had elapsed. The bar was removed from the chamber during this phase for this group, and the opening was covered with a piece of aluminum matching the wall of the chamber. The generalization control group, C-GEN, received presentations of the light in the same manner that Group R-S* experienced the tone. Finally, a no-treatment control group, C-No, was placed in the chamber with the bar out and neither the light nor the tone presented. Each rat had two sessions during this phase, each lasting $30 \mathrm{~min}$. Groups $\mathrm{S}_{\mathrm{T}^{-}} \mathrm{S}^{*}, \mathrm{R}-\mathrm{S}^{*}$, and C-Gen received a total of 120 presentations of the appropriate stimulus (the trial postponement for intertrial responses was not in effect for Group S-S*), each coming $30 \mathrm{sec}$ after the previous presentation.

In Phase 3, all groups were treated identically. The bar was in the chamber, and the tone was presented. Responses terminated the tone and operated the empty pellet dispenser. If no response occurred in $10 \mathrm{sec}$ after the presentation of the tone, the tone terminated and the empty pellet dispenser operated. The data for extinction are the mean number of responses to the tone per 5-min interval, averaged across the two $30-\mathrm{min}$ sessions during this phase. In addition, intertrial responses (between tone presentations) were also recorded.

\section{RESULTS}

Two dependent variables will be reported: responses to the discrete signal and intertrial responses.

\section{Phase 1}

The rats quickly learned to press the bar in the presence of either the light or the tone. On Session 4 (the first with the final contingency in effect), the mean number of trials of light and tone presentation was 28.10, and on Session 13, it was 67.68. Since trials were separated by a minimum of $20 \mathrm{sec}$ in the absence of intertrial responding, and assuming $5 \mathrm{sec}$ was needed for a barpress and eating the food pellet, the theoretical maximum number of trials per session was 72 . By the end of training, the subjects were thus very efficient in their performance. The analysis of variance on the four groups for Sessions 4 and 13 revealed a significant sessions effect $[F(1,28)=265.55, p<.01]$ and nonsignificant differences due to groups $[F(3,28)=.59]$ and the interaction of groups and sessions $[F(3,28)=.70]$.

\section{Phase 2}

Only the S-S* group had the bar present during this phase of the experiment, so the only data were the extinction of barpressing to the light (and intertrial responses) for this group. The mean total number of responses to the light on the last session of this phase was 13.3. Most of these responses occurred in the first 5 min of the session (mean $=4.6$ ), and by the last $5 \mathrm{~min}$, the mean number of responses was .7. Thus for this group, extinction of responding to the light was fairly substantial, as intended.

\section{Phase 3}

The mean number of responses to the tone for each group is shown in Figure 1. As can be seen, the two control groups made more responses to the tone than 


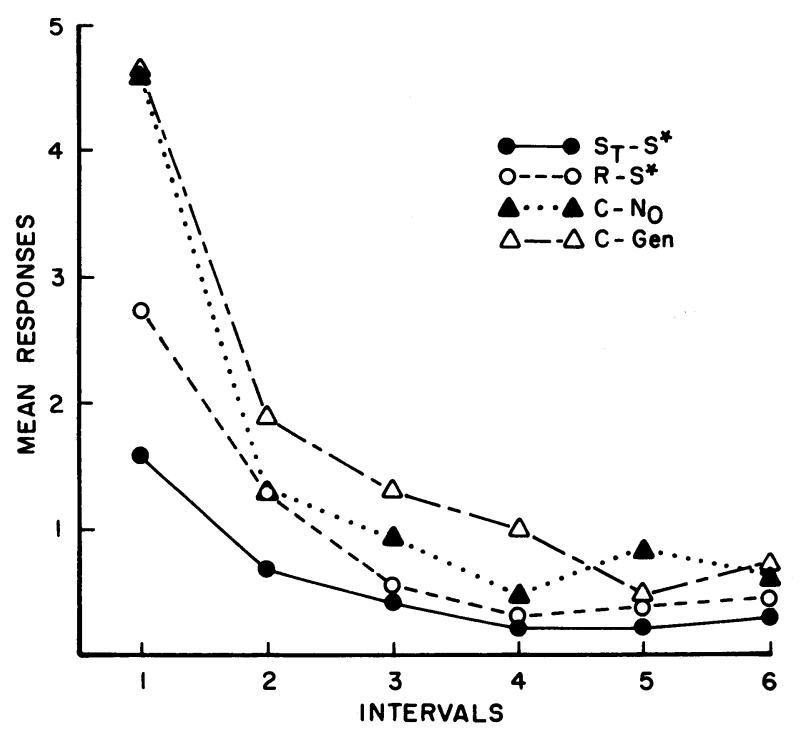

Figure 1. Mean number of tone-terminating responses averaged across two extinction sessions in 5-min intervals.

did the other two experimental groups, particularly in the first 5-min interval of extinction. Importantly, the group $\left(\mathrm{S}_{\mathrm{T}}-\mathrm{S}^{*}\right)$ that had the response to the light extinguished in Phase 2 was less resistant to extinction than the group (R-S*) that had the tone-food association extinguished in Phase 2.

An analysis of variance on groups (four) by intervals (six) by sessions (two) was performed and confirmed the effects that are evident in Figure 1. The number of responses to the tone differed significantly between groups $[F(3,28)=3.68, p<.03]$, and the Groups by Intervals interaction was also significant $[F(15,140)=$ $2.72, \mathrm{p}<.01]$. Of course, the main effect for intervals was significant $[F(5,140)=50.17, p<.01]$, confirming the rather obvious decline in responding across the 30 min of extinction, and the main effect for sessions was also significant $[F(1,28)=13.97, p<.01]$, confirming what is not shown in Figure 1, that responding decreased from the first to the second extinction session. The sessions factor did not interact significantly with any of the other factors.

The significant Groups by Intervals interaction justified the comparison of groups using Tukey's 1.s.d. method for post hoc comparisons. On the first interval, the two control groups were not significantly different from each other, but they were superior to Group R-S* and Group $\mathrm{S}_{\mathrm{T}}-\mathrm{S}^{*}$. Importantly, Group $\mathrm{S}_{\mathrm{T}}-\mathrm{S}^{*}$ (R-S* association extinguished) was significantly less resistant to extinction than Group R-S* (the $\mathrm{S}_{\mathrm{T}}-\mathrm{S}^{*}$ association extinguished). A difference of .77 between points on Figure 1 is need for the .05 level of significance using the 1.s.d. comparison procedure. Thus the $\mathrm{S}_{\mathrm{T}}-\mathrm{S}^{*}$ association supported less responding in extinction than did the R-S* association. The fact that both groups showed significantly less responding to the tone than did the control groups indicates that R-S* and $\mathrm{S}_{\mathrm{T}}-\mathrm{S}^{*}$ each contribute to the persistence of responding, even though their contributions are not equal in magnitude.

The effects of the Phase 2 extinction procedures were specific to the extinction of responses to the tone. This is shown by the lack of difference between groups in intertrial responses during Phase 3. The analysis of intertrial responses showed a significant effect of intervals $[F(5,140)=12.22, p<.01]$, with most of the intertrial responses occurring during the first two 5-min intervals. Differences attributable to groups $[F(3,28)=$ .97] and interactions of groups with intervals or days were not significant. Thus the effects of the various extinction treatments during Phase 3 were specific to the tone-barpress-food associative process and did not produce generalized effects, at least as evidenced by intertrial responding.

\section{DISCUSSION}

These data indicate that both a Pavlovian, S-S* association and an operant or instrumental R-S* association are effective in determining the persistence of barpress responding by rats. This finding is particularly important in the context of theories that rely exclusively on the $\mathrm{S}_{-} \mathrm{S}^{*}$ association as the explanatory mechanism for operant behavior (e.g., Bindra, 1974, 1978). In fact, the present study suggests that extinction of the R-S* association may have a more decremental effect on persistence than extinction of the S-S* association.

Given that it is our contention that an understanding of operant behavior must take into account some degree of learning about the response, it is important to consider the possibility that our presumed weakening of the R-S* association actually had an effect on the response and is not analyzable in terms of the weakening of an S-S* association. In particular, it might be argued that extinction of the response in the presence of the light might weaken the association between the cues associated with approach and contact with the barpress mechanism and food. According to this argument, the effect of extinguishing the response to the light would not be a direct weakening of an R-S* association but would actually weaken an S-S* association in which the " $S$ " includes the cues accompanying approach to and contact with the barpress mechanism. The loss of incentive value for these stimuli would account for the effectiveness of extinguishing responding to the light. However, if the incentive value for such stimuli was affected, then it would seem that there might have been some effect on intertrial responses during Phase 3. Since approach and contact cues have been extinguished, we would expect a reduced tendency of the $S_{T^{-}} S^{*}$ rats to make intertrial responses compared with the other groups, and in fact no significant difference was present.

Even more damaging to the view that the S-S* associative process is the dominant influence in determining the persistence of operant behavior is the finding that extinction of the tonefood association was not as effective in reducing responding to the tone as was extinction of the response-food association. Even though the barpress mechanism was removed from the operant box for the group experiencing tone/no-food trials, presumably, many of the cues that would be assumed to be part of an S-S* association governing approach and contact with the bar would still be present, and their incentive value would be lost. If an S-S* mechanism is to carry the explanatory burden of operant behavior, then it appears that Group R-S* should have been the least resistant to extinction, and in fact, Group S-S* was the least resistant. 
Another type of S-S* mechanism to be considered is the possibility that the response is actually governed by an S-S* association with the $\mathrm{S}$ representing subject-generated proprioceptive stimuli. The present procedure does not allow us to rule out the possibility that extinction or weakening of the R-S* association actually is a weakening of an S-S* association with the $S$ representing proprioceptive cues. Indeed, response learning may mean that subject-generated stimuli have entered into an association with an $\mathrm{S}^{*}$. If this is the case, then the only question left is whether S-S* associations involving endogenous stimuli are subject to the same laws as S-S* associations involving exogenous stimuli.

Finally, the fact that extinction of responding to the light (Group $\mathrm{S}_{\mathrm{T}-} \mathrm{S}^{*}$ ) had a large effect would seem to indicate that a three-term associative mechanism, S(R-S*), cannot explain the results. Such a three-term mechanism is much like Skinner's original conceptualization of operant learning in which a discriminative stimulus sets the occasion for the reinforcement of a response, and the structure of this three-term mechanism is not represented by the simple combination of S-S* and R-S* association (e.g., Jenkins, 1977). The fact that extinction of responding to the light resulted in very marked reduction of responding to the tone indicates that the control of responding under one stimulus is not independent of the control under a different stimulus. If the subjects had learned separate $S_{T}\left(R-S^{*}\right)$ and $S_{\mathrm{L}}\left(\mathrm{R}-\mathrm{S}^{*}\right)$ associations, it would seem that Group $\mathrm{S}_{\mathrm{T}}-\mathrm{S}^{*}$ should not have differed from the control groups, and clearly, there was a difference.

\section{REFERENCES}

Amsel, A. Partial reinforcement effects on vigor and persistence. In K. W. Spence \& J. T. Spence (Eds.), The psychology of learning and motivation (Vol. 1). New York: Academic Press, 1967.

Bindra, D. A. A motivational view of learning, performance, and behavior modification. Psychological Review, 1974, 81, 199-213.

Bindra, D. How adaptive behavior is produced: A perceptualmotivational alternative to response-reinforcement. Behavioral and Brain Sciences, 1978, 1, 41-91.

Brown, P. L., \& Jenkins, H. M. Auto-shaping of the pigeon's key peck. Journal of the Experimental Analysis of Behavior, $1968,11,1-8$.

Capaldi, E. J. A sequential hypothesis of instrumental learning. In K. W. Spence \& J. T. Spence (Eds.), The psychology of learning and motivation (Vol. 1). New York: Academic Press, 1967.

Jenkins, H. M. Sensitivity of different response systems to stimulus-reinforcer and response-reinforcer relations. In $\mathrm{H}$. Davis \& H. M. B. Hurwitz (Eds.), Operant-Pavlovian interactions. Hillsdale, N.J: Erlbaum, 1977.

Hearst, E., \& Jenkins, H. M. Sign tracking: The stimulusreinforcer relation and directed action. Austin, Tex: Psychonomic Society, 1974.

Moore, B. R. The role of directed Pavlovian reactions in simple instrumental learning in the pigeon. In R. A. Hinde \& J. Stevenson-Hinde (Eds.), Constraints on learning. London: Academic Press, 1973.

(Received for publication August 17, 1980.) 